\title{
Analysis of Bacterial Biofilms on a Cochlear Implant Following Methicillin-Resistant Staphylococcus Aureus Infection
}

\author{
Gi Jung $\mathrm{Im}^{1}$, Yun Suk An ${ }^{2}$, June Choi ${ }^{1}$, Jae Jun Song ${ }^{1}$, Sung Won Chae ${ }^{1}$, and Hak Hyun Jung ${ }^{1}$ \\ ${ }^{1}$ Department of Otolaryngology-Head and Neck Surgery, Korea University College of Medicine, Seoul, \\ ${ }^{2}$ Department of Otolaryngology-Head and Neck Surgery, Daejin Medical Center, Yeoju, Korea
}

\author{
Received August 17, 2015 \\ Revised October 21, 2015 \\ Accepted November 4, 2015
}

To demonstrate biofilm formations on a cochlear implant magnet of a pediatric patient suffering from a methicillin-resistant Staphylococcus aureus (MRSA) infection. The appearance of biofilm colonies was analyzed on different magnet sections. The appearance of MRSA biofilms on the surface of an explanted cochlear implant was analyzed by scanning electron microscopy (SEM), focusing on the pattern of extracellular polymeric substances (EPS) within the biofilms. SEM revealed unique biofilms with a three-dimensional EPS complex and tower-like formations. Biofilm configurations changed from the margin to the center of the magnet. Biofilms were solitary and scattered at the margin; large and plate-like in the center; and stacked in layers, forming towers and water channels, in the middle region. After a MRSA infection, biofilm formations were observed on the surface of a magnet. Bacterial biofilms provide optimal conditions for bacterial growth and antibiotic resistance and can cause intractable infections that lead to device failure. J Audiol Otol 2015;19(3):172-177

KEY WORDS: Biofilm · Scanning electron microscopy · Extracellular polymeric substance Cochlear implant · Methicillin-resistant Staphylococcus aureus.

\section{Introduction}

Postoperative infection after cochlear implant (CI) surgery is somewhat rare, occurring in only 1.7 to $3.3 \%$ of the population [1]. Wound care to prevent infection of the $\mathrm{CI}$ is critical for implanted patients because infectious complications frequently involve skin flap necrosis, wound dehiscence, persistent swelling, and potential device extrusion. The most common organism isolated from CI infections is Staphylococcus aureus (73.3\%, 11/15 cases) [1]. Methicillin-resistant Staphylococcus aureus (MRSA) is resistant to multiple antibiotics and can be a common cause of persistent infection in CI patients. MRSA and Pseudomonas species frequently cause biofilms on implants, and these bacterial biofilm formations can reduce the susceptibility of microorganisms to antibiotics [2].

A biofilm is an organic entity consisting of a complex of mi-

This is an Open Access article distributed under the terms of the Creative Commons Attribution Non-Commercial License (http://creativecommons. org/licenses/by-nc/3.0/) which permits unrestricted non-commercial use, distribution, and reproduction in any medium, provided the original work is properly cited. crobial colonies adhering to a three-dimensional matrix made up of extracellular polymeric substances (EPS) [3]. Biofilms attach to inert implant surfaces and can facilitate bacterial growth and survival. Thus, biofilms are an important consideration in CI management. First, the bacteria can be latent because the requirements for oxygen and nutrients are reduced in biofilms, and waste products are easily disposed of through countless water channels [4]. Second, the formation of biofilms enhances the antibiotic resistance of resident bacteria, and biofilms on CIs can result in persistent infection and inflammation [5]. Third, the artificial implant surface plays an essential role in the establishment of bacterial biofilms. Biofilms are most substantial in depressions along the surface of devices, and similar accumulations have been observed in depressions of the electrodes and CI magnet $[3,6]$.

In the present study, we demonstrate the presence of biofilms on an explanted CI of a pediatric patient suffering from a MRSA infection. We describe the three-dimensional formations of biofilms on the removable magnet of the $\mathrm{CI}$ and analyze the morphological pattern of biofilm colonies on different magnet sections. 


\section{Case Report}

\section{Case history}

A 28-month-old child presented to the local otolaryngology clinic for a cochlear implantation. She had been diagnosed with a profound hearing loss at the age of 18 months and used hearing aids, which were reportedly not helpful. The cochlear implantation was performed successfully on the right ear, and a prophylactic antibiotic (second-generation cephalosporin) was intravenously administered before the procedure. Three weeks after implantation, the patient was responsive to sound using the CI. But there were but swelling and redness in the inferior portion of the posterior auricular incision. The lesion was a stitch abscess with a $1 \times 1 \mathrm{~cm}^{2}$ sized pustule and a piece of absorbable Vicryl (Ethicon, Inc., Somerville, NJ, USA) in its center. Initially, a second-generation cephalosporin was intravenously administered along with local, topical applications of ciprofloxacin ointment. Four weeks after the implantation, a granulation tissue appeared on the inferior portion of the incision. Although the lesion was small and localized, the purulent area persisted even after the intensive topical therapy. MRSA was found in the intensive culture, and systemic vancomycin was added to the treatment regimen. The granulation tissue was about $1 \mathrm{~cm}$ in diameter and was coated with exudate. Since the wound failed to heal after 6 weeks of topical and systemic antibiotic therapy, the patient underwent surgery for wound debridement. The stimulator-receiver of the implant was washed with saline, and the infected periosteum was excised. Then the wound was closed with a scalp rotation flap. However, 1 week later, the area around the CI receiver began to swell again. After treatment with an oral corticosteroid and intravenous antibiotics, the acute inflammation was controlled. Nevertheless, wound swelling was repeated and sustained, and complete eradication of the infection did not appear to be possible. Three months after the patient's debridement, the skin covering the $\mathrm{CI}$ became thinner, and a portion of the implant was exposed (Fig. 1). Consequently, we decided to remove the device. The CI electrode array did not appear to be involved in the infection and was left in the cochlea to prevent fibrous or osseous obliteration of the scala tympani. Surprisingly, the wound healed immediately after implant removal. A few months later, a cochlear implantation was performed on the left ear, and the outcome of the procedure and the auditory results were excellent. One year after CI extraction, a new CI device was inserted in the previously infected side, and the cochlear implantation was successful. At that time, the remnant CI electrode was extracted and analyzed by scanning electron microscopy (SEM).

\section{Scanning electron microscopy}

The magnet unit was removed from the CI device, rinsed with phosphate-buffered saline (PBS), transferred to Trump's solution, and transported to the electron microscopy core laboratory for preparation. The sample was washed three times for 10 minutes with PBS and once for 10 minutes with distilled water. The sample was then dehydrated in a graded ethanol series of $25 \%, 50 \%, 75 \%, 95 \%$, and $100 \%$ for 10 minutes at each step. After two 5-minute washes with hexamethyldisilazane, the sample was dried in a vacuum desiccator. Finally, the sample was attached to a beveled disk and coated with palladium and gold using an argon gas sputter coating unit. The external surface of the magnet, consisting of hollow tubes, was scanned with a Hitachi S-4000 scanning electron microscope (Tokyo, Japan) with a magnification range of $\times 30$ to $\times 15000$. Images were taken at an accelerator voltage of $6 \mathrm{kV}$ and were digitally recorded. Qualitative analysis of the images was performed.
Fig. 1. Postaural skin defect and electrode exposure in a patient with a cochlear implant $(\mathrm{Cl})$ on the right side. A: The $\mathrm{Cl}$ device was infected with methicillin-resistant Staphylococcus aureus. Swelling around the device and electrode exposure was observed. B: Massive fibrosis and purulent discharge were observed upon $\mathrm{Cl}$ device extraction. Severe adhesions were seen in the infected device and electrode.
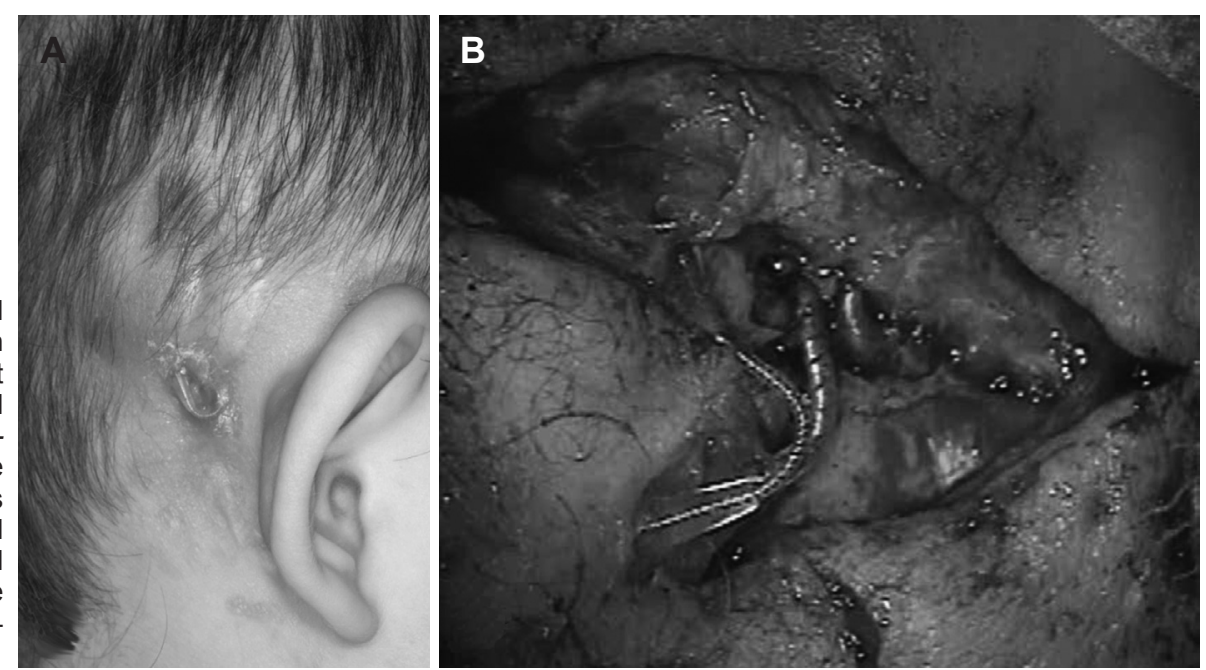


\section{Scattered biofilms at the margin of the magnet}

At the margin of the magnet, there were many scattered biofilms, which appeared as isolated forms or soft tissue masses (Fig. 2). Biofilms at the margin of the magnet did not show tower-like forms or water channels. The surface of the magnet appeared uneven, bumpy, and rough when viewed under high magnification $(\times 2000)$. These irregular surfaces may make initial attachment of biofilms much easier.
Plate-like and mature biofilms at the magnet center

In the central region of the magnet, large and plate-like biofilms were observed, and these biofilms frequently overlapped (overlapping planks of biofilms). The surface of the plate-like biofilms appeared smooth with a few cracks. Complex towerlike three-dimensional structures were observed under high magnification (Fig. 3). Under the plate-like outer wall of mature biofilms, these tower-like three-dimensional structures
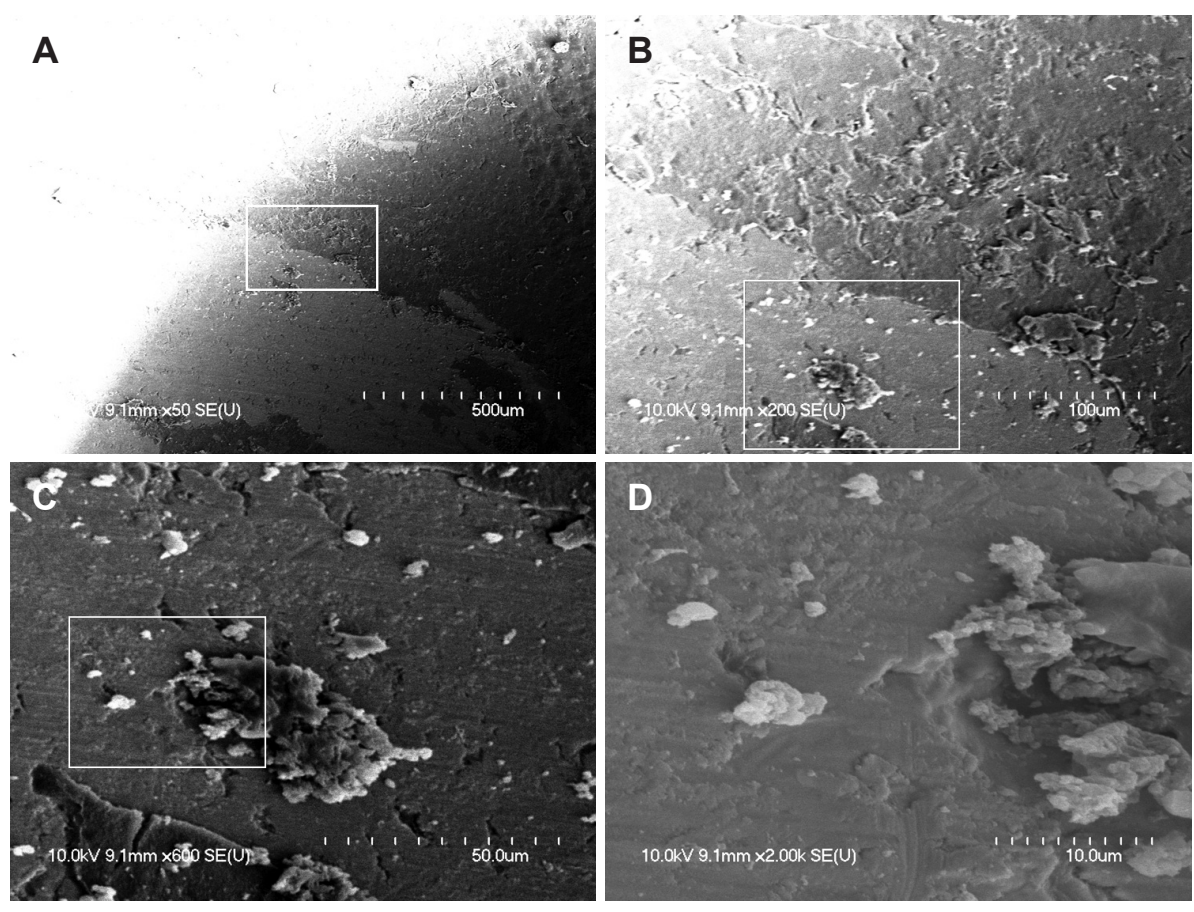

Fig. 2. A: Left upper periphery of the cochlear implant $(\mathrm{Cl})$ magnet showing scattered clusters of biofilms ( $\times 50)$. B, C: Many isolated and solitary biofilm clusters showing initial attachment $(\times 200, \times 600)$. D: Biofilm displaying a three-dimensional structure and attached to the irregular surface of the $\mathrm{Cl}$ magnet $(x$ 2000). The region in the white box is magnified consecutively from $A$ to $D$.
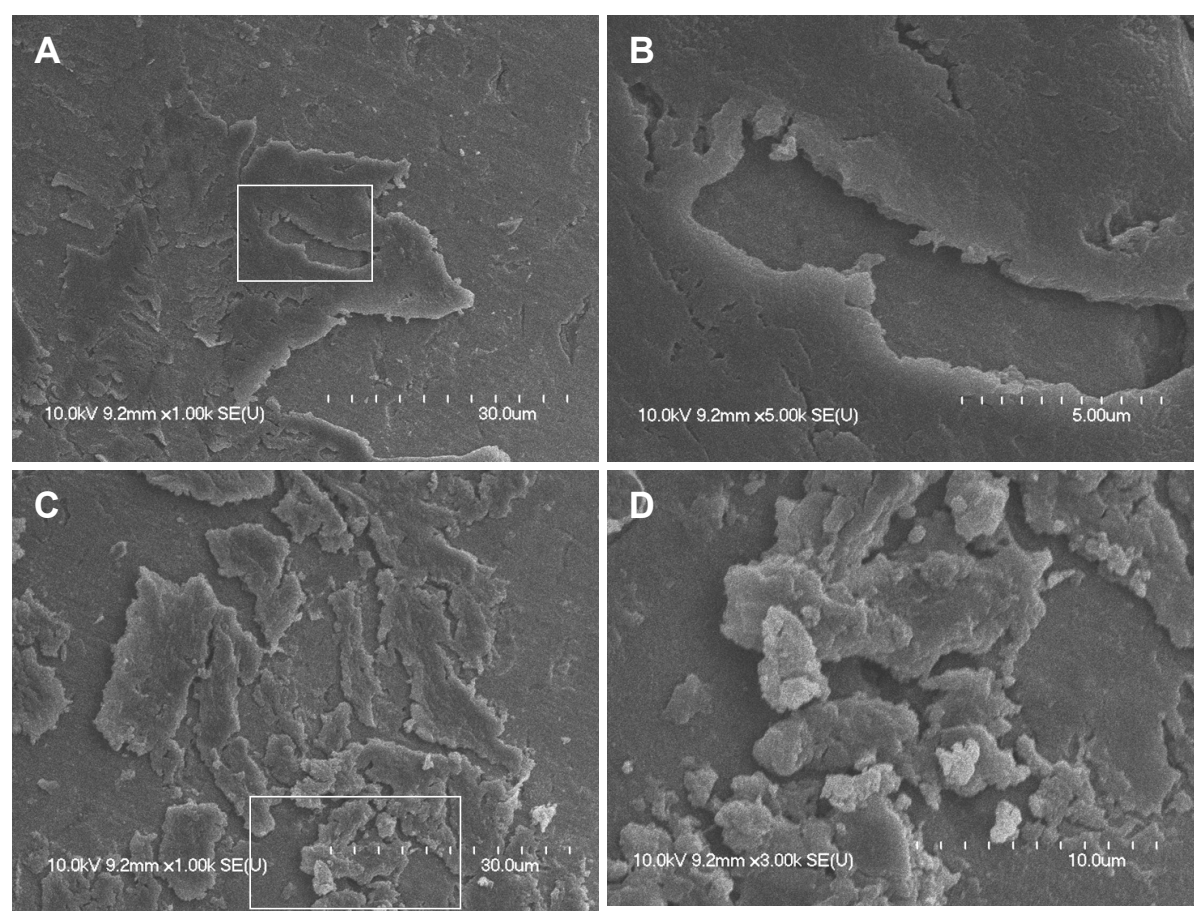

Fig. 3. A, B: Large, plate-like biofilms forming three-dimensional structures around a large cavity and taking up much of the magnet surface $(\times 1000$, $\times 5000$ ). The surface of the platelike biofilms appeared smooth. C, D: Plate-like biofilms were cracked or scattered, and many biofilm clusters of various sizes were observed. Complex tower-like three-dimensional structures were observed in the cracks $(\times 1000, \times 5000)$. The region in the white box is magnified from $A$ to $B$ and from $C$ to $D$. 
formed numerous holes and water channels.

\section{Biofilm colonies forming towers and water channels in the middle region}

In the middle region between the margin and the center of the magnet, countless clusters of biofilms showed typical growth phases in which colonization and formation of a polysaccharide matrix were observed (Fig. 4). Biofilms were well attached to the irregular surface of the magnet. Biofilm structures were stacked in layers that contained many holes and water channels, which could have provided optimal conditions for bacterial growth.

\section{Comparison of biofilm coverage among magnet sections}

Analysis of SEM images revealed that biofilms covered $7 \pm$ $5 \%, 51 \pm 10.5 \%$, and $69 \pm 13.5 \%$ of the margin, middle re-
Fig. 4. A: Countless clusters of biofilms showing typical growth phases in which colonization and polysaccharide matrix formation occur $(\times 1000)$. B: Biofilms were well attached to the irregular surface of the magnet. Biofilms grew and matured to form towers stacked in layers $(x$ 5000). C: Biofilm clusters showing evidence of stacked towers and water channels $(\times 15000)$. D: Under the cracked plate-like biofilms, tower-like three-dimensional structures formed numerous holes and water channels $(\times 15000)$. The region in the white $d$ box is magnified consecutively from $A$ to $C$ and $D$.
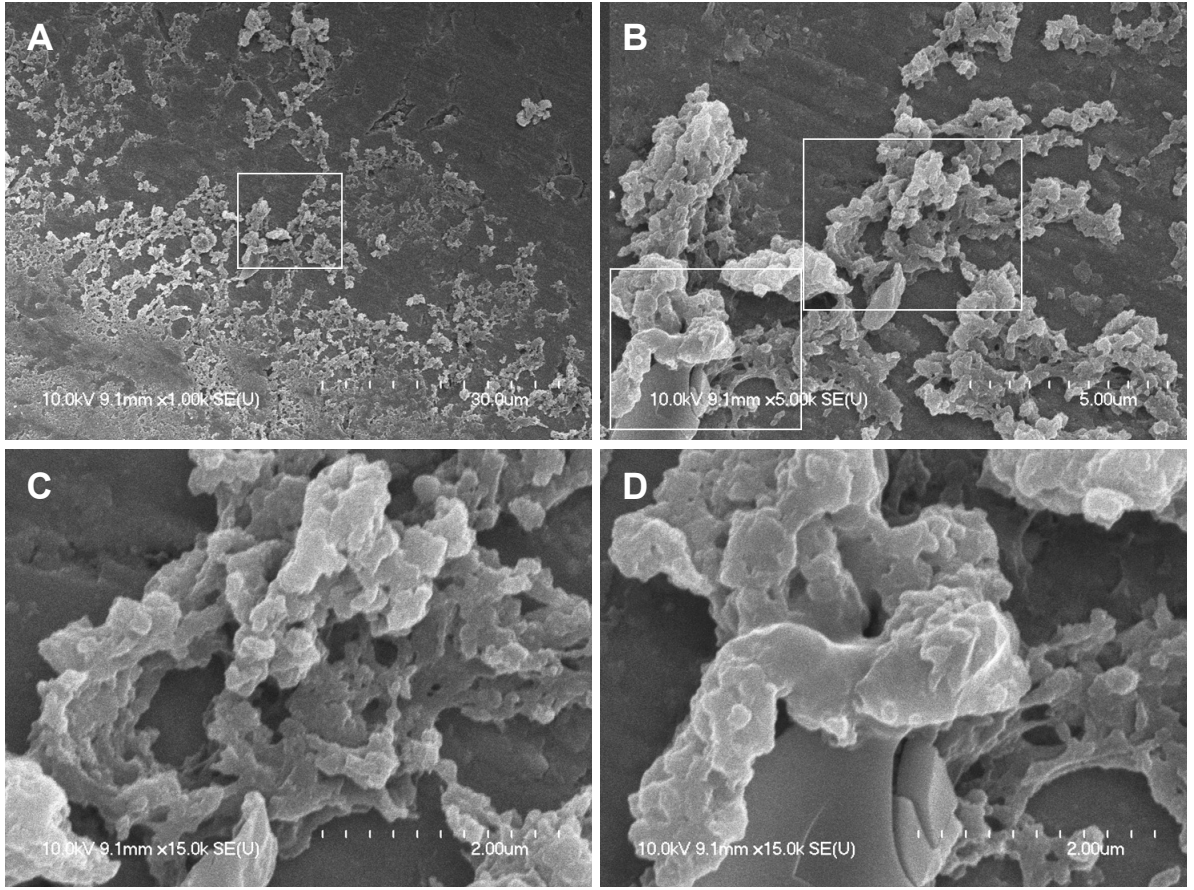
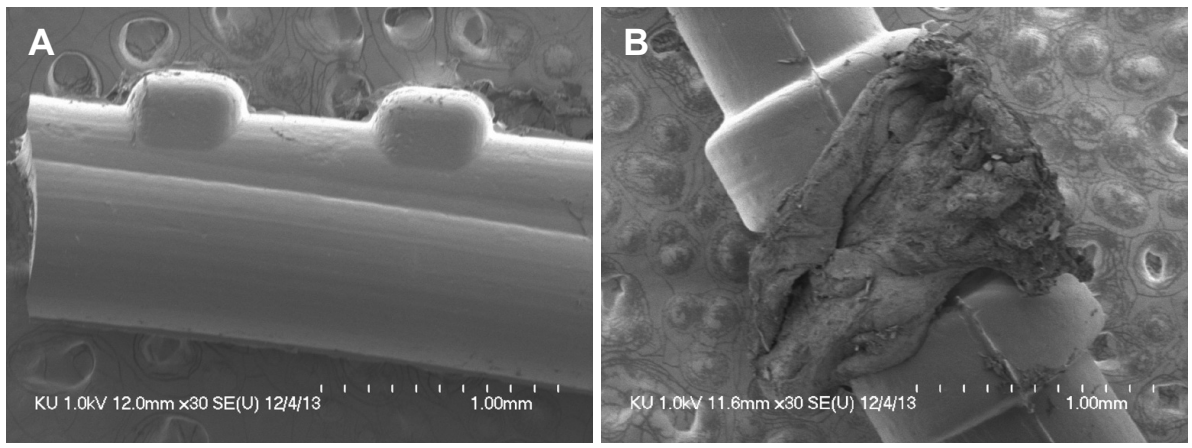

Fig. 5. The electrode of the cochlear implant $(\mathrm{Cl})$ was left in the scala tympani of the cochlea to prevent cochlear ossification when the infected $\mathrm{Cl}$ device was extracted. At the time of cochlear reimplantation, the remnant $\mathrm{Cl}$ electrode was extracted and analyzed using scanning electron microscopy. There were no biofilm formations in the proximal part of the electrode $(A)$, the cochleostomy site (B), the mid-portion of the electrode (C), or the distal part of the electrode (D).
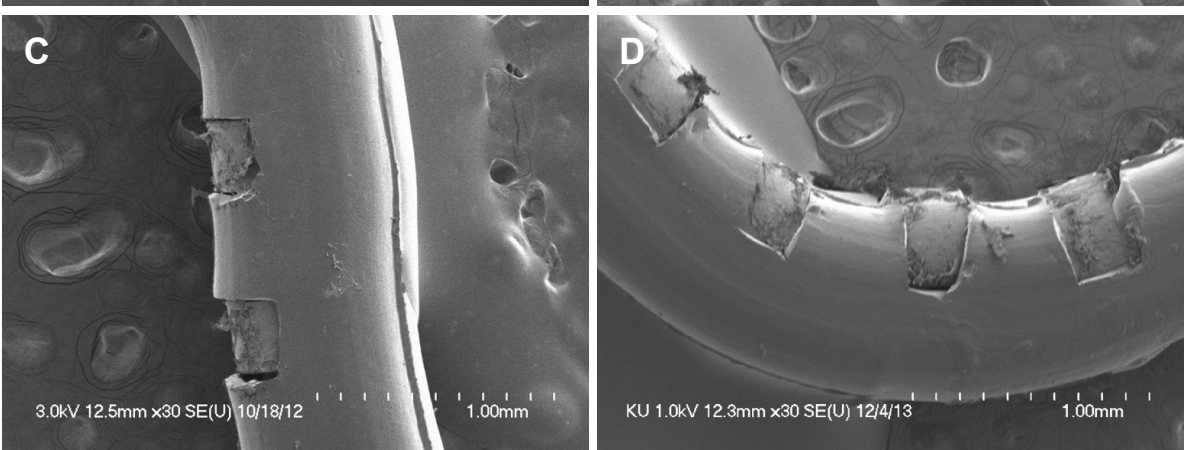
gion, and center of the magnet, respectively $(n=5)$. One-way analysis of variance with Tukey's post hoc analysis was used to compare biofilm-covered areas among sections. The biofilmcovered area increased significantly $(p<0.05)$ from the margin of the magnet to the center, indicating that the growth of biofilms in the center of the magnet was significantly greater than that in the marginal area. This difference in biofilm growth may have been due to a difference in external exposure.

\section{Remnant electrode free of biofilms}

When the new CI was implanted in the previously infected side, the remnant $\mathrm{CI}$ electrode was extracted and analyzed by SEM (Fig. 5). There were no biofilms on the proximal part of the electrode (Fig. 5A), the cochleostomy site (Fig. 5B), the midportion of the electrode (Fig. $5 \mathrm{C}$ ), or the distal part of the electrode (Fig. 5D).

\section{Discussion}

Our study addresses the presence of biofilms in human CIs after MRSA infection. Specifically, this study suggests that biofilms are a possible cause of implant failure, especially after a MRSA infection. The patient described in this report had been suffering from frequent swelling and inflammation, despite massive doses of antibiotics. There are two possible ways in which biofilms can cause device failure. First, biofilms may protect bacteria from antibiotics, resulting in a latent and persistent infection. Second, it is possible that the biofilm causes an allergic reaction, resulting in persistent inflammation. In either case, it is evident that biofilms play an important role in postsurgical infection and device failure. Third, adverse effects of biofilms include facilitation of bacterial metabolism and growth, blockage of antibiotic penetration, and increased resistance to antibiotics [7].

In this study, differences in biofilm appearance were observed from the periphery to the center of the CI magnet. Little biofilm existed in the peripheral area of the magnet, presumably because this area was partially covered by silicone. In contrast, the center of the magnet, located below the periosteum of the skull, was exposed externally, so MRSA biofilm could have formed more readily in this region. In fully mature biofilm, the outer wall formed a plate-like barrier. This plate-like appearance represents a unique pattern of over-maturation, because biofilm on a $\mathrm{CI}$ is in a confined space and cannot detach or disseminate.

We did not find any biofilms on the remnant $\mathrm{CI}$ electrode in the previously infected side. Therefore, when extracting an infected CI device, it is safe and appropriate to leave the CI electrode in the scala tympani of the cochlea to prevent cochlear ossification, even in cases of MRSA infection.

Despite prolonged therapy with vancomycin and massive debridement with irrigation, MRSA-infected CIs will most likely have to be removed in nearly all cases [8]. Such aggressive virulence is due to MRSA-specific molecular changes that facilitate mechanisms such as quorum sensing, adhesion, bacteriophage mobilization, exotoxin production, intracellular persistence, and biofilm formation, all of which contribute to a more severe infection [2]. It is surprising that even after irrigation of the infected CI device, the three-dimensional EPS complex remained on the surface of the magnet. The rate of MRSA infection has been increasing because of the inappropriate use of antibiotics, which not only encourages overgrowth of MRSA but may also enhance pathogenicity. The potential synergy between biofilm formation and MRSA infection seems to cause serious sequelae in CI patients. For the surgical management of MRSA-infected CI patients, we recommend replacement of the $\mathrm{CI}$ magnet and massive irrigation of the CI device. Rotational flap surgery is another treatment option, but destructive or not cosmetic for children.

In CI operation, seroma-free operation is essential to prevent potential infection. Thus, meticulous care should be required as below, 1) proper position of CI on smooth skull surface not protruding or no pressure on the scalp, 2) tight perosteal flap closure to compress $\mathrm{CI}, 3$ ) skin glue and sterile strip to avoid stitch abscess, 4) $\mathrm{H}_{2} \mathrm{O}_{2}$ dressing to destroy biofilms on operative preparations.

Biofilms begin to form after initial attachment to a surface, including tissue surfaces. After host matrix proteins adequately cover an abiotic surface, biofilms can grow and mature much faster [9]. The irregular surfaces of CIs, especially the indented area of the magnet, may make this initial attachment of biofilms much easier, so further preventative measures should be taken, including coating devices or magnets with antibiotics or an inert material [10].

Although this SEM analysis of a MRSA-infected CI provided valuable information, the study had some limitations. First, biofilms were observed only on the CI magnet, not the entire CI device. Second, SEM requires a dehydration process, which can fossilize the matrix architecture and reduce the population of live bacteria. However, this study showed clear views of the three-dimensional EPS matrix and different growth patterns on different regions of the magnet. Further studies should focus on a device coated with a biofilm-resistant material and on salvage treatment of a MRSA-infected device.

In an explanted $\mathrm{CI}$ of a pediatric patient suffering from a MRSA infection, SEM demonstrated unique biofilms and a three-dimensional EPS complex. After a MRSA infection, biofilm formations were observed on the surface of a CI mag- 
net, especially the central depressed area. Bacterial biofilms can cause intractable infections that lead to device failure and extraction. Biofilm patterns also changed from the margin to the center, with solitary and scattered biofilm forms in the margin, large and plate-like formations in the center, and stacked layers forming towers and water channels in the middle region. This study provides insight into biofilms and the EPS complex on CIs, which provide optimal conditions for bacterial growth and antibiotic resistance.

\section{Acknowledgments}

This research was supported by the Basic Science Research Program through the National Research Foundation of Korea (NRF) funded by the Ministry of Education (NRF-2014-071497;R1429731) and by the Technology Innovation Program (10049743, Establishing a medical device development open platform, as a hub for excellerating close firm-hospital communication) funded by the Ministry of Trade, industry \& Energy (MI, Korea).

\section{REFERENCES}

1) Cunningham CD 3rd, Slattery WH 3rd, Luxford WM. Postoperative infection in cochlear implant patients. Otolaryngol Head Neck Surg 2004;131:109-14.

2) Dancer SJ. The effect of antibiotics on methicillin-resistant Staphylococcus aureus. J Antimicrob Chemother 2008;61:246-53.

3) Pawlowski KS, Wawro D, Roland PS. Bacterial biofilm formation on a human cochlear implant. Otol Neurotol 2005;26:972-5.

4) Sanclement JA, Webster P, Thomas J, Ramadan HH. Bacterial biofilms in surgical specimens of patients with chronic rhinosinusitis. Laryngoscope 2005;115:578-82.

5) Antonelli PJ, Lee JC, Burne RA. Bacterial biofilms may contribute to persistent cochlear implant infection. Otol Neurotol 2004;25:953-7.

6) Loeffler KA, Johnson TA, Burne RA, Antonelli PJ. Biofilm formation in an in vitro model of cochlear implants with removable magnets. Otolaryngol Head Neck Surg 2007;136:583-8.

7) Lewis K. Riddle of biofilm resistance. Antimicrob Agents Chemother 2001;45:999-1007.

8) Brady AJ, Farnan TB, Toner JG, Gilpin DF, Tunney MM. Treatment of a cochlear implant biofilm infection: a potential role for alternative antimicrobial agents. J Laryngol Otol 2010;124:729-38.

9) Otto M. Staphylococcal biofilms. Curr Top Microbiol Immunol 2008; 322:207-28.

10) Jang CH, Park H, Cho YB, Choi CH. Effect of vancomycin-coated tympanostomy tubes on methicillin-resistant Staphylococcus aureus biofilm formation: in vitro study. J Laryngol Otol 2010;124:594-8. 